\title{
Health Diagnosing and Treating Practitioners
}

National Cancer Institute

\section{Source}

National Cancer Institute. Health Diagnosing and Treating Practitioners. NCI Thesaurus. Code C122465.

The group of professions that include chiropractors, dentists, dietitians and nutritionists, optometrists, pharmacists, physicians and surgeons, physicians assistants, podiatrists, registered nurses, therapists, and veterinarians. 